\title{
Optimal channel switching in multiuser systems under average capacity constraints
}

\author{
Ahmet Dundar Sezer, Sinan Gezici* \\ Department of Electrical and Electronics Engineering, Bilkent University, Bilkent, Ankara 06800, Turkey
}

\section{A R T I C L E I N F O}

\section{Article history:}

Available online 20 January 2017

\section{Keywords:}

Channel switching

Capacity

Multiuser

Time sharing

Power allocation

\begin{abstract}
A B S T R A C T
In this paper, the optimal channel switching problem is studied for average capacity maximization in the presence of multiple receivers in the communication system. First, the optimal channel switching problem is proposed for average capacity maximization of the communication between the transmitter and the secondary receiver while fulfilling the minimum average capacity requirement of the primary receiver and considering the average and peak power constraints. Then, an alternative equivalent optimization problem is provided and it is shown that the solution of this optimization problem satisfies the constraints with equality. Based on the alternative optimization problem, it is obtained that the optimal channel switching strategy employs at most three communication links in the presence of multiple available channels in the system. In addition, the optimal strategies are specified in terms of the number of channels employed by the transmitter to communicate with the primary and secondary receivers. Finally, numerical examples are provided in order to verify the theoretical investigations.
\end{abstract}

(C) 2017 Elsevier Inc. All rights reserved.

\section{Introduction}

Optimal power allocation has critical importance for enhancing performance of communication systems. For example, in fading environments, performance of communication between two users can be improved by employing an efficient power allocation strategy (e.g., water-filling algorithm [1]) compared to the conventional uniform power allocation approach. In the literature, the studies related to power allocation have mostly focused on the performance metrics such as channel capacity (e.g., [1-3]), bit error rate (BER) (e.g., [4-8]), and outage probability (e.g., [9-11]) in general. In [1], the optimal power allocation strategy is derived for capacity maximization over a fading additive white Gaussian noise (AWGN) channel in the presence of perfect channel state information (CSI) at both the transmitter and the receiver. It is obtained that the optimal strategy that maximizes the channel capacity is the waterfilling solution in which more power is allocated to better channel states if the signal-to-noise ratio (SNR) is above a certain threshold and no power is transmitted otherwise. Via optimal power alloca-

\footnotetext{
This research was supported in part by the Distinguished Young Scientist Award of Turkish Academy of Sciences (TUBA-GEBIP 2013). A.D. Sezer is also supported by ASELSAN Graduate Scholarship for Turkish Academicians.

* Corresponding author. Fax: +90 3122664192.

E-mail addresses: adsezer@ee.bilkent.edu.tr (A.D. Sezer), gezici@ee.bilkent.edu.tr (S. Gezici).
}

tion, the ergodic capacity and the outage capacity is maximized in [2] for secondary users in a cognitive radio network. In a similar context, the optimal power allocation schemes are considered in [4] for cognitive radio networks in order to minimize the average BER of secondary users. In [9], the optimal power allocation is studied in order to reduce the outage probability in fading channels.

In addition to the power allocation approach, time sharing (i.e., randomization) is another method for improving performance of communication systems. The mechanism behind the benefits of the time sharing (randomization) method is related to a phenomenon called stochastic resonance (SR). The counterintuitive effects of SR provides performance benefits in the context of statistical average for a system in which nonlinearities and suboptimal parameters are observed $[12,13]$. In the literature, the time sharing approach has been studied in the context of noise enhanced detection and estimation (e.g., [14-18]), error performance improvement (e.g., [16,19-24]), and jamming performance enhancement (e.g., [25-27]). Although an increase in the noise degrades the system performance in general, addition of noise to a system in conjunction with time sharing among a certain number of signal levels can provide performance benefits [14-18]. In a similar context, stochastic signaling, i.e., time sharing among multiple signal values for each information symbol, is performed for average power constrained non-Gaussian channels to improve the error performance of the system [19,20]. In [19], it is presented that randomization 
(time sharing) is required among no more than three different signal values in order to achieve the optimal error performance in the presence of second and fourth moment constraints. Also, time sharing among multiple detectors (i.e., detector randomization) is employed over additive time-invariant noise channels [16,21]. In [16], it is obtained that time sharing between two antipodal signal pairs and the corresponding maximum a-posteriori probability (MAP) detectors can significantly enhance the system performance in the presence of symmetric Gaussian mixture noise. In a similar manner, the study in [21] investigates both detector randomization and stochastic signaling approaches for an $M$-ary communication system in which an additive noise channel is considered with a known distribution. In the context of jamming performance enhancement, a jammer can employ time sharing among multiple power levels in order to reduce the detection performance of a receiver or to degrade the error performance of a communication system [25-27].

In the presence of multiple channels in a communication system, time sharing (i.e., channel switching) can be employed to enhance certain performance metrics such as average probability of error, average number of correctly received symbols, and channel capacity [28-31]. The channel switching problem is studied in [28] for $M$-ary communication systems in which a transmitter communicates with a receiver by employing a stochastic signaling approach in order to minimize the average probability of error under an average power constraint. It is shown that the optimal strategy corresponds to either one of the following strategies: deterministic signaling over a single channel, time sharing between two different signal constellations over a single channel, or time sharing between two channels with deterministic signaling over each channel. The channel switching problem is also studied in [29] for maximizing the average number of correctly received symbols between a transmitter and a receiver in the presence of average power and cost constraints. It is proved that the optimal strategy corresponds to channel switching either among at most three different channels with full channel utilization (i.e., no idle periods), or between at most two different channels with partial channel utilization. Unlike the studies in [28] and [29], the channel switching strategy is employed together with power allocation in order to enhance the capacity of a communication system in [30,31]. In [30], the optimal channel switching strategies are investigated for a communication system in which a single transmitter communicates with a single receiver in the presence of the average and peak power constraints. It is obtained that the optimal channel switching strategy corresponds to the exclusive use of a single channel or to channel switching between two channels. In [31], the study in [30] is extended for a communication system where the channel switching delays (costs) are considered due to hardware limitations. It is shown that any channel switching strategy consisting of more than two different channels cannot be optimal.

Although the channel switching problem has been studied for communication between a single transmitter and a single receiver in the presence of average and peak power constraints and in the consideration of channel switching delays, no studies in the literature have considered the channel switching problem in the presence of multiple receivers in the communication system. In this study, a transmitter communicates with two receivers (classified as primary and secondary) by employing a channel switching strategy among available multiple channels in the system. The aim of the transmitter is to enhance the average capacity of the secondary receiver while satisfying the minimum average capacity requirement for the primary receiver in the presence of average and peak power constraints. ${ }^{1}$ Also, due to hardware limitations, the transmitter can establish only one communication link with one of the receivers at a given time by employing one of the communication channels available in the system. It is obtained that if more than one channel is available, then the optimal channel switching strategy which maximizes the average capacity of the secondary receiver consists of no more than 3 communication links. (It is important to note that each channel in the system constitutes two communication links; that is, one for the communication between the transmitter and the primary receiver and one for the communication between the transmitter and the secondary receiver.) In addition, with regard to the number of channels employed in the optimal channel switching strategy, it is concluded that the transmitter either communicates with the primary receiver over at most two channels and employs a single channel for the secondary receiver, or communicates with the primary receiver over a single channel and employs at most two channels for the secondary receiver. In addition to the communication system with a single primary receiver, the channel switching problem in this study is also extended for communication systems in which there exist multiple primary receivers, each having a separate minimum average capacity requirement for the communication with the transmitter. Lastly, numerical examples are provided to exemplify the theoretical results.

Compared to this manuscript, the studies in [30] and [31] do not consider the multi-user scenario and consequently the optimal channel strategies obtained in those studies are not applicable for a communication system in which multiple users communicate with each other. Even though the studies in [30] and [31] do not provide any approaches for multi-user communication systems, they constitute a fundamental aspect for the optimal channel switching strategies obtained in this manuscript. Therefore, the methods and approaches employed in this study bear a certain level of resemblance to those in [30] and [31]. On the other hand, it is important to note that the contributions of this study to the literature are significantly different from the ones in [30] and [31]. More precisely, the constraint related to the minimum average capacity requirement of the primary receiver in the communication system modeled in this study alters the analysis of the optimal channel switching strategy and requires new proof approaches that are mostly different from the ones employed in [30] and [31].

The main contributions of this paper can be summarized as follows:

- For the first time in the literature, the channel switching problem is studied for average capacity maximization in the presence of multiple receivers in a communication system where the transmitter communicates with the primary and secondary receivers in order to improve the average capacity of the secondary receiver under the average and peak power constraints and the minimum average capacity requirement for the primary receiver.

- It is obtained that the optimal channel switching strategy includes no more than 3 communication links in the presence of multiple available communication channels in the system.

- It is shown that the optimal channel switching strategy corresponds to one of the following strategies:

- The transmitter performs communication with the primary receiver over at most two channels and employs a single channel for the secondary receiver.

\footnotetext{
1 In this study, the channel switching delays are omitted in order to simplify the system model. However, the main contributions of the manuscript are valid in the presence of switching delays, as well.
} 


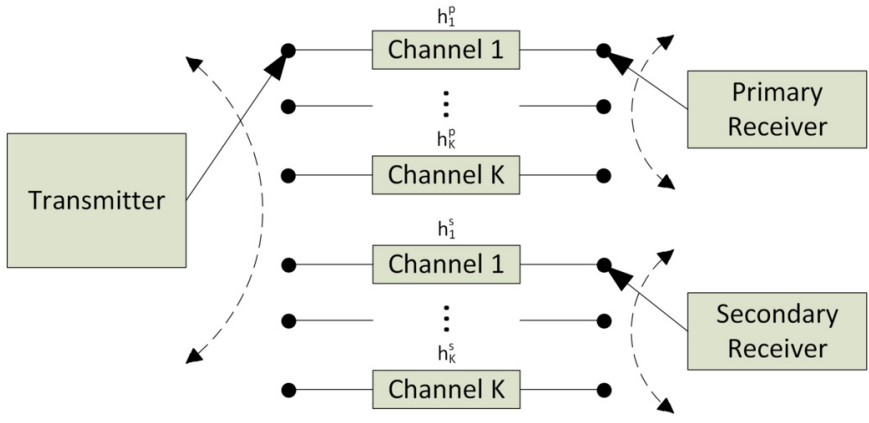

Fig. 1. Block diagram of a communication system in which transmitter communicates with primary and secondary receivers via channel switching among $K$ channels (frequency bands). It is noted that the channel coefficients can be different for the same channels.

- The transmitter communicates with the primary receiver over a single channel and at most two channels are occupied for the communication to the secondary receiver.

- A low-complexity solution to the channel switching problem is provided, which requires the comparison of the average capacities obtained by two optimization problems, each having significantly lower computational complexity than the original channel switching problem.

- As an extension, the channel switching problem is reformulated in the consideration of multiple primary receivers and their corresponding minimum average capacity requirements.

\section{System model and problem formulation}

Consider a communication system in which $K$ different channels (frequency bands) are available for a transmitter to communicate with two receivers classified as primary and secondary. ${ }^{2}$ It is assumed that, due to hardware constraints, the transmitter can establish only one communication link with one of the receivers at a given time by performing communication over one of the channels [30,31]. The reason for this assumption is that the transmitter and the receivers are assumed to have a single RF chain each due to complexity and cost considerations. The restriction caused by this assumption simplifies the circuit and antenna design at transmitters and receivers while reducing the hardware costs by allowing to employ a single RF chain to transmit/receive data. The transmitter can switch (time share) among these $K$ channels to improve the average capacity of the secondary receiver while satisfying the minimum average capacity requirement for the primary receiver. The channels are modeled as statistically independent flat-fading additive Gaussian noise channels with constant power spectral density levels over the channel bandwidths. Also, the channel state information (CSI) is assumed to be available at both the transmitter and the associated receiver, and the channels can have different bandwidths and constant spectral density levels in general. Fig. 1 illustrates the system model with $K$ different channels (frequency bands), where the transmitter communicates with one primary and one secondary receiver via channel switching (i.e., time sharing). In practice, the transmitter can initiate communication with the primary receiver and communicate over one channel for a certain fraction of time. Then, it switches to another channel and communicates with the primary receiver over that channel for another fraction of time. The similar pro-

\footnotetext{
2 Extensions to multiple receivers are presented in Section 4. Also, the terms, primary and secondary, used in the study have different meanings from the ones used in the cognitive radio literature where primary users are licensed users and secondary users are unlicensed users that are allowed to access the spectrum when primary users are not active.
}

cess continues for the remaining channels. Later, the transmitter establishes communication with the secondary receiver and it applies the same procedure as employed for the primary receiver; that is, for a certain fraction of time, it communicates with the secondary transmitter over one channel and it switches to the remaining channels in order and communicates over those channels for certain fractions of time. It is important to emphasize that the receivers are classified as primary and secondary in the study since the transmitter primarily satisfies the minimum average capacity requirement for the primary receiver and then performs communication with the secondary receiver to enhance the average capacity of the communication with the secondary receiver. This scenario is applicable to wireless sensor networks in which child nodes can employ the channel switching strategy in order to improve their average capacity while fulfilling the minimum average capacity constraint of the parent node. Also, it can be stated that the channel switching strategy may improve the energy efficiency of the communication system by requiring a lower average power to achieve the same average channel capacity achieved by the conventional methods [32,33].

Let $B_{i}$ and $N_{i} / 2$ denote, respectively, the bandwidth and the constant power spectral density level of the additive Gaussian noise for channel $i$, where $i \in\{1, \ldots, K\}$, and let $h_{i}^{k}$ represent the complex channel gain for channel $i$ between the transmitter and receiver $k$, where $k \in\{\mathrm{p}, \mathrm{s}\}$ denotes the label for either the primary or the secondary receiver. Then, the capacity of channel $i$ between the transmitter and receiver $k$ is expressed as

$C_{i}^{k}(P)=B_{i} \log _{2}\left(1+\frac{\left|h_{i}^{k}\right|^{2} P}{N_{i} B_{i}}\right)$ bits/sec

where $P$ represents the average transmit power [34].

The main objective of this study is to determine the optimal channel switching strategy that maximizes the average capacity of the communication between the transmitter and the secondary receiver while ensuring the minimum average capacity constraint for the primary receiver with the consideration of average and peak power constraints. To provide a mathematical formulation, time-sharing (channel switching) factors are defined as $\lambda_{1}^{\mathrm{p}}, \ldots, \lambda_{K}^{\mathrm{p}}, \lambda_{1}^{\mathrm{s}}, \ldots, \lambda_{K}^{\mathrm{s}}$, where $\lambda_{i}^{\mathrm{p}}$ and $\lambda_{i}^{\mathrm{s}}$ denote the fractions of time when channel $i$ is utilized by the transmitter for communication with the primary receiver and the secondary receiver, respectively. Then, the following optimal channel switching problem is proposed for average capacity maximization of the link between the transmitter and the secondary receiver under a minimum average capacity constraint of the primary receiver:

$$
\begin{aligned}
\max _{\left\{\lambda_{i}^{\mathrm{p}}, \lambda_{i}^{\mathrm{s}}, P_{i}^{\mathrm{p}}, P_{i}^{s}\right\}_{i=1}^{K}} & \sum_{i=1}^{K} \lambda_{i}^{\mathrm{s}} C_{i}^{\mathrm{s}}\left(P_{i}^{\mathrm{s}}\right) \\
\text { subject to } & \sum_{i=1}^{K} \lambda_{i}^{\mathrm{p}} C_{i}^{\mathrm{p}}\left(P_{i}^{\mathrm{p}}\right) \geq C_{\text {req }} \\
& \sum_{i=1}^{K}\left(\lambda_{i}^{\mathrm{p}} P_{i}^{\mathrm{p}}+\lambda_{i}^{\mathrm{s}} P_{i}^{\mathrm{s}}\right) \leq P_{\mathrm{av}}, \\
& P_{i}^{\mathrm{p}}, P_{i}^{\mathrm{s}} \in\left[0, P_{\mathrm{pk}}\right], \forall i \in\{1, \ldots, K\} \\
& \sum_{i=1}^{K}\left(\lambda_{i}^{\mathrm{p}}+\lambda_{i}^{\mathrm{s}}\right)=1, \\
& \lambda_{i}^{\mathrm{p}}, \lambda_{i}^{\mathrm{s}} \in[0,1], \forall i \in\{1, \ldots, K\}
\end{aligned}
$$

where $C_{i}^{k}\left(P_{i}\right)$ for $k \in\{\mathrm{p}, \mathrm{s}\}$ is as in (1), $P_{i}^{\mathrm{p}}$ and $P_{i}^{\mathrm{s}}$ represent the average transmit powers allocated to channel $i$ in order to communicate with the primary and secondary receivers, respectively, 
$C_{\text {req }}$ is the minimum average capacity requirement for the primary receiver, $P_{\mathrm{pk}}$ denotes the peak power limit, and $P_{\mathrm{av}}$ represents the average power limit for the transmitter. The average power limit can be associated with the power consumption and/or the battery life at the transmitter. On the other hand, the peak power constraint refers to the maximum power level that can be produced by the transmitter circuitry (i.e., a hardware constraint). It is assumed that $P_{\mathrm{av}}<P_{\mathrm{pk}}$ and $C_{\mathrm{req}}>0$. It is also important to note that there exists a total of $2 K$ communication links in the system since each of the $K$ channels (frequency bands) can be used for communicating with the primary receiver or secondary receiver.

\section{Optimal channel switching for communication between the transmitter and the secondary receiver}

Since the optimization problem in (2) is not convex and requires a search over a $4 K$ dimensional space in general, it is hard to obtain the solution of the problem in its current form. Therefore, the aim is to convert the optimization problem in (2) into a tractable equivalent optimization problem, the solution of which is the same as that of (2). The following optimization problem represents such an alternative optimization problem.

Proposition 1. The following optimization problem results in the same maximum average capacity for the secondary receiver as the original optimization problem in (2):

$$
\begin{aligned}
\max _{\left\{\lambda_{i}^{\mathrm{p}}, \lambda_{i}^{\mathrm{s}}, P_{i}^{\mathrm{p}}, P_{i}^{\mathrm{s}}\right\}_{i=1}^{K}} & \sum_{i=1}^{K} \lambda_{i}^{\mathrm{s}} C_{\max }^{\mathrm{s}}\left(P_{i}^{\mathrm{s}}\right) \\
\text { subject to } & \sum_{i=1}^{K} \lambda_{i}^{\mathrm{p}} C_{\max }^{\mathrm{p}}\left(P_{i}^{\mathrm{p}}\right) \geq C_{\text {req }} \\
& \sum_{i=1}^{K}\left(\lambda_{i}^{\mathrm{p}} P_{i}^{\mathrm{p}}+\lambda_{i}^{\mathrm{s}} P_{i}^{\mathrm{s}}\right) \leq P_{\mathrm{av}}, \\
& P_{i}^{\mathrm{p}}, P_{i}^{\mathrm{s}} \in\left[0, P_{\mathrm{pk}}\right], \forall i \in\{1, \ldots, K\} \\
& \sum_{i=1}^{K}\left(\lambda_{i}^{\mathrm{p}}+\lambda_{i}^{\mathrm{s}}\right)=1, \\
& \lambda_{i}^{\mathrm{p}}, \lambda_{i}^{\mathrm{s}} \in[0,1], \forall i \in\{1, \ldots, K\}
\end{aligned}
$$

where $C_{\max }^{k}(P)$ is defined as

$C_{\text {max }}^{k}(P) \triangleq \max \left\{C_{1}^{k}(P), \ldots, C_{K}^{k}(P)\right\}$

for $k \in\{\mathrm{p}, \mathrm{s}\}$.

Proof. Let $\left\{\tilde{\lambda}_{i}^{\mathrm{p}}, \tilde{\lambda}_{i}^{\mathrm{s}}, \tilde{P}_{i}^{\mathrm{p}}, \tilde{P}_{i}^{\mathrm{s}}\right\}_{i=1}^{K}$ denote the solution of the optimization problem in (2) and $C^{*}$ denote the corresponding maximum average capacity. Then, the achieved maximum average capacity for the communication between the transmitter and the secondary receiver can be written as $C^{*}=\sum_{i=1}^{K} \tilde{\lambda}_{i}^{s} C_{i}^{s}\left(\tilde{P}_{i}^{s}\right)$. From the definition of $C_{\max }^{k}$ in (4), the following relation is obtained:

$C^{*}=\sum_{i=1}^{K} \tilde{\lambda}_{i}^{s} C_{i}^{s}\left(\tilde{P}_{i}^{s}\right) \leq \sum_{i=1}^{K} \tilde{\lambda}_{i}^{s} C_{\max }^{s}\left(\tilde{P}_{i}^{s}\right)$.

It is noted that $\left\{\tilde{\lambda}_{i}^{\mathrm{p}}, \tilde{\lambda}_{i}^{\mathrm{s}}, \tilde{P}_{i}^{\mathrm{p}}, \tilde{P}_{i}^{\mathrm{s}}\right\}_{i=1}^{K}$ satisfies the constraints in (3). Therefore, it is deduced that the problem in (3) can achieve the maximum average capacity obtained by the problem in (2); that is, $C^{*} \leq C^{\star}$, where $C^{\star}$ denotes the maximum average capacity according to (3). Next, consider the solution of the optimization problem in (3). The maximum average capacity obtained by (3) can be expressed as $C^{\star}=\sum_{i=1}^{K} \bar{\lambda}_{i}^{\mathrm{s}} C_{\max }^{\mathrm{s}}\left(\bar{P}_{i}^{\mathrm{s}}\right)$, where $\left\{\bar{\lambda}_{i}^{\mathrm{p}}, \bar{\lambda}_{i}^{\mathrm{s}}, \bar{P}_{i}^{\mathrm{p}}, \bar{P}_{i}^{\mathrm{s}}\right\}_{i=1}^{K}$ denotes the solution of (3). Now, define functions $g^{(k)}(i)$ for $k \in\{\mathrm{p}, \mathrm{s}\}$ and sets $S_{m}^{(k)}$ for $k \in\{\mathrm{p}, \mathrm{s}\}$ as follows ${ }^{3}$ :

$$
g^{(k)}(i) \triangleq \underset{l \in\{1, \ldots, K\}}{\arg \max } C_{l}\left(\bar{P}_{i}^{k}\right), \forall i \in\{1, \ldots, K\}
$$

and

$S_{m}^{(k)} \triangleq\left\{i \in\{1, \ldots, K\} \mid g^{(k)}(i)=m\right\}, \forall m \in\{1, \ldots, K\}$.

Then, the following relations can be obtained for $k \in\{\mathrm{p}, \mathrm{s}\}$ :

$$
\begin{aligned}
\sum_{i=1}^{K} \bar{\lambda}_{i}^{k} C_{\max }^{k}\left(\bar{P}_{i}^{k}\right) & =\sum_{i=1}^{K} \bar{\lambda}_{i}^{k} C_{g^{(k)}(i)}^{k}\left(\bar{P}_{i}^{k}\right) \\
& =\sum_{i=1}^{K} \sum_{n \in S_{i}^{(k)}} \bar{\lambda}_{n}^{k} C_{i}^{k}\left(\bar{P}_{n}^{k}\right) \\
& \leq \sum_{i=1}^{K}\left(\sum_{n \in S_{i}^{(k)}} \bar{\lambda}_{n}^{k}\right) C_{i}^{k}\left(\frac{\sum_{n \in S_{i}^{(k)}} \bar{\lambda}_{n}^{k} \bar{P}_{n}^{k}}{\sum_{n \in S_{i}^{(k)}} \bar{\lambda}_{n}^{k}}\right) \\
& =\sum_{i=1}^{K} \hat{\lambda}_{i}^{k} C_{i}^{k}\left(\hat{P}_{i}^{k}\right)
\end{aligned}
$$

where $\hat{\lambda}_{i}^{k}$ and $\hat{P}_{i}^{k}$ are defined as

$\hat{\lambda}_{i}^{k} \triangleq \sum_{n \in S_{i}^{(k)}} \bar{\lambda}_{n}^{k}$ and $\hat{P}_{i}^{k} \triangleq \frac{\sum_{n \in S_{i}^{(k)}} \bar{\lambda}_{n}^{k} \bar{P}_{n}^{k}}{\sum_{n \in S_{i}^{(k)}} \bar{\lambda}_{n}^{k}}$

for $i \in\{1, \ldots, K\}$. The equalities in (8) and (9) are obtained from the definitions in (6) and (7), respectively, and the inequality in (10) follows from Jensen's inequality due to the concavity of the capacity function $[34,35]$. Based on the inequality in (8)-(11), it is obtained that $\hat{\lambda}_{i}^{\mathrm{p}}$ 's and $\hat{P}_{i}^{\mathrm{p}}$ 's satisfy the minimum average capacity requirement in (2); that is, $\sum_{i=1}^{K} \hat{\lambda}_{i}^{\mathrm{p}} C_{i}^{\mathrm{p}}\left(\hat{P}_{i}^{\mathrm{p}}\right) \geq C_{\text {req }}$ since $\sum_{i=1}^{K} \hat{\lambda}_{i}^{\mathrm{p}} C_{i}^{\mathrm{p}}\left(\hat{P}_{i}^{\mathrm{p}}\right) \geq \sum_{i=1}^{K} \bar{\lambda}_{i}^{\mathrm{p}} C_{\max }^{\mathrm{p}}\left(\bar{P}_{i}^{\mathrm{p}}\right)$ and $\sum_{i=1}^{K} \bar{\lambda}_{i}^{\mathrm{p}} C_{\max }^{\mathrm{p}}\left(\bar{P}_{i}^{\mathrm{p}}\right) \geq$ $C_{\text {req. }}$. Also, it is noted from (12), based on (6) and (7), that $\hat{\lambda}_{i}^{k}$ 's and $\hat{P}_{i}^{k}$, s for $k \in\{\mathrm{p}, \mathrm{s}\}$ satisfy the other constraints in (2); that is, $\sum_{i=1}^{K}\left(\hat{\lambda}_{i}^{\mathrm{p}} \hat{P}_{i}^{\mathrm{p}}+\hat{\lambda}_{i}^{\mathrm{s}} \hat{P}_{i}^{\mathrm{s}}\right) \leq P_{\mathrm{av}}, \hat{P}_{i}^{\mathrm{p}}, \hat{P}_{i}^{\mathrm{s}} \in\left[0, P_{\mathrm{pk}}\right], \forall i \in\{1, \ldots, K\}$, $\sum_{i=1}^{K}\left(\hat{\lambda}_{i}^{\mathrm{p}}+\hat{\lambda}_{i}^{s}\right)=1$, and $\hat{\lambda}_{i}^{\mathrm{p}}, \hat{\lambda}_{i}^{s} \geq 0, \forall i \in\{1, \ldots, K\}$. Therefore, the inequality in (8)-(11), namely, $C^{\star} \leq \sum_{i=1}^{K} \hat{\lambda}_{i} C_{i}\left(\hat{P}_{i}\right)$, implies that the optimal solution of (3) cannot achieve a higher average capacity than that achieved by $(2)$; that is, $C^{\star} \leq C^{*}$. Hence, it is concluded that $C^{\star}=C^{*}$ since $C^{\star} \geq C^{*}$ must also hold as mentioned at the beginning of the proof.

Based on Proposition 1, the solution of the original problem in (2) can be obtained from the optimization problem in (3), which is more tractable than the one in (2), as investigated in the following. Proposition 1 also implies that an optimal strategy always utilizes the best channel for a given power level, as noted from (3a), (3b), and (4), which is intuitive due to the monotone increasing nature of the capacity expression.

As a first step towards characterizing the solution of (3), the following proposition provides a useful statement that the constraints in (3b) and (3c) always hold with equality.

\footnotetext{
3 In the case of multiple maximizers in (6), any maximizing index can be chosen for $g^{(k)}(i)$.
} 
Proposition 2. The solution of the optimization problem in (3) satisfies the constraints in (3b) and (3c) with equality; that is, $\sum_{i=1}^{K} \bar{\lambda}_{i}^{\mathrm{p}} C_{\max }^{\mathrm{p}}\left(\bar{P}_{i}^{\mathrm{p}}\right)$ $=C_{\text {req }}$ and $\sum_{i=1}^{K} \bar{\lambda}_{i}^{\mathrm{p}} \bar{P}_{i}^{\mathrm{p}}+\bar{\lambda}_{i}^{\mathrm{s}} \bar{P}_{i}^{\mathrm{s}}=P_{\mathrm{av}}$, where $\left\{\bar{\lambda}_{i}^{\mathrm{p}}, \bar{\lambda}_{i}^{\mathrm{s}}, \bar{P}_{i}^{\mathrm{p}}, \bar{P}_{i}^{s}\right\}_{i=1}^{K}$ denotes the solution of (3).

Proof. Assume that $\left\{\bar{\lambda}_{i}^{\mathrm{p}}, \bar{\lambda}_{i}^{\mathrm{s}}, \bar{P}_{i}^{\mathrm{p}}, \bar{P}_{i}^{\mathrm{s}}\right\}_{i=1}^{K}$ is the solution of (3) such that $\sum_{i=1}^{K}\left(\bar{\lambda}_{i}^{\mathrm{p}} \bar{P}_{i}^{\mathrm{p}}+\bar{\lambda}_{i}^{\mathrm{s}} \bar{P}_{i}^{\mathrm{s}}\right)<P_{\mathrm{av}}$. Then, the following cases are considered $^{4}$ :

- If $\bar{\lambda}_{i}^{s}=0, \forall i \in\{1, \ldots, K\}$, then there exists at least one $\bar{P}_{i}^{\mathrm{p}}$ such that $\bar{P}_{i}^{\mathrm{p}}<P_{\mathrm{pk}}$ since $\sum_{i=1}^{K} \lambda_{i}^{\mathrm{p}} P_{i}^{\mathrm{p}} \leq P_{\mathrm{av}}$ and $\sum_{i=1}^{K} \lambda_{i}^{\mathrm{p}}=1$ due to the constraints in (3c) and (3d), respectively, and $P_{\mathrm{av}}<P_{\mathrm{pk}}$ by the assumption for (2). Let $\bar{P}_{l}^{\mathrm{p}}$ denote one of them. Then, consider an alternative solution $\left\{\hat{\lambda}_{i}^{\mathrm{p}}, \hat{\lambda}_{i}^{\mathrm{s}}, \hat{P}_{i}^{\mathrm{p}}, \hat{P}_{i}^{\mathrm{s}}\right\}_{i=1}^{K}$, where

$\hat{P}_{l}^{\mathrm{p}}=\min \left\{P_{\mathrm{pk}}, \bar{P}_{l}^{\mathrm{p}}+\left(P_{\mathrm{av}}-\sum_{i=1}^{K} \bar{\lambda}_{i}^{\mathrm{p}} \bar{P}_{i}^{\mathrm{p}}\right) / \bar{\lambda}_{l}^{\mathrm{p}}\right\}$,

$\hat{\lambda}_{l}^{\mathrm{p}}=\frac{\bar{\lambda}_{l}^{\mathrm{p}} C_{\max }^{\mathrm{p}}\left(\bar{P}_{l}^{\mathrm{p}}\right)}{C_{\max }^{\mathrm{p}}\left(\hat{P}_{l}^{\mathrm{p}}\right)}$,

$\hat{\lambda}_{i}^{\mathrm{p}}=\bar{\lambda}_{i}^{\mathrm{p}}, \forall i \in\{1, \ldots, K\} \backslash\{l\}$,

$\hat{P}_{i}^{\mathrm{p}}=\bar{P}_{i}^{\mathrm{p}}, \forall i \in\{1, \ldots, K\} \backslash\{l\}$,

$\hat{\lambda}_{1}^{\mathrm{s}}=\bar{\lambda}_{l}^{\mathrm{p}}-\hat{\lambda}_{l}^{\mathrm{p}}$,

$\hat{P}_{1}^{\mathrm{s}}=\hat{P}_{l}^{\mathrm{p}}$,

$\hat{\lambda}_{i}^{s}=\bar{\lambda}_{i}^{s}, \forall i \in\{2, \ldots, K\}$,

$\hat{P}_{i}^{\mathrm{s}}=\bar{P}_{i}^{\mathrm{s}}, \forall i \in\{2, \ldots, K\}$.

The solution $\left\{\bar{\lambda}_{i}^{\mathrm{p}}, \bar{\lambda}_{i}^{\mathrm{s}}, \bar{P}_{i}^{\mathrm{p}}, \bar{P}_{i}^{\mathrm{s}}\right\}_{i=1}^{K}$ achieves an average capacity of $\bar{C}^{s}=0$ due to $\bar{\lambda}_{i}^{s}=0, \forall i \in\{1, \ldots, K\}$. On the other hand, the alternative solution satisfies the constraints in (3) and achieves a larger capacity; that is $\hat{C}^{s}=\hat{\lambda}_{1}^{s} C_{\max }^{s}\left(\hat{P}_{1}^{s}\right)>0$ since $\hat{\lambda}_{1}^{s}>0$ and $\hat{P}_{1}^{\mathrm{s}}>0$. Therefore, $\left\{\bar{\lambda}_{i}^{\mathrm{p}}, \bar{\lambda}_{i}^{\mathrm{s}}, \bar{P}_{i}^{\mathrm{p}}, \bar{P}_{i}^{\mathrm{s}}\right\}_{i=1}^{K}$ cannot be optimal if $\bar{\lambda}_{i}^{s}=0, \forall i \in\{1, \ldots, K\}$, which contradicts with the assumption at the beginning of the proof.

- For the case that $\bar{\lambda}_{i}^{s}>0, \exists i \in\{1, \ldots, K\}$, define a set as

$M \triangleq\left\{i \in\{1, \ldots, K\} \mid \bar{\lambda}_{i}^{s}>0\right\}$.

Next, consider the following cases:

- If $\bar{P}_{k}^{\mathrm{s}}=P_{\mathrm{pk}}, \forall k \in M$, then there exists at least one $\bar{P}_{i}^{\mathrm{p}}$ that satisfies $\bar{P}_{i}^{\mathrm{p}}<P_{\mathrm{pk}}$ since the constraints in (3c) and (3d) hold. Let $\bar{P}_{l}^{\mathrm{p}}$ represent one of them and consider an alternative solution $\left\{\hat{\lambda}_{i}^{\mathrm{p}}, \hat{\lambda}_{i}^{\mathrm{s}}, \hat{P}_{i}^{\mathrm{p}}, \hat{P}_{i}^{\mathrm{s}}\right\}_{i=1}^{K}$, where $\hat{P}_{l}^{\mathrm{p}}, \hat{\lambda}_{l}^{\mathrm{p}}, \hat{\lambda}_{i}^{\mathrm{p}}$ for all $i \in\{1, \ldots, K\} \backslash\{l\}, \hat{P}_{i}^{\mathrm{p}}$ for all $i \in\{1, \ldots, K\} \backslash\{l\}, \hat{\lambda}_{1}^{\mathrm{s}}$, and $\hat{P}_{1}^{\mathrm{s}}$ are as in (13)-(18) and the remaining terms are as follows:

$$
\begin{aligned}
& \hat{\lambda}_{2}^{\mathrm{s}}=\sum_{k \in M} \bar{\lambda}_{k}^{\mathrm{s}}, \\
& \hat{P}_{2}^{\mathrm{s}}=P_{\mathrm{pk}}, \\
& \hat{\lambda}_{i}^{\mathrm{s}}=0, \forall i \in\{3, \ldots, K\}, \\
& \hat{P}_{i}^{\mathrm{s}}=0, \forall i \in\{3, \ldots, K\} .
\end{aligned}
$$

\footnotetext{
4 In this case, it is assumed that multiple channels are available for communication; that is, $K>1$. In the case of a single channel available for communication (i.e., $K=1$ ), a similar approach can be employed.
}

The achieved average capacity by $\left\{\bar{\lambda}_{i}^{\mathrm{p}}, \bar{\lambda}_{i}^{\mathrm{s}}, \bar{P}_{i}^{\mathrm{p}}, \bar{P}_{i}^{\mathrm{s}}\right\}_{i=1}^{K}$ is $\bar{C}^{s}=$ $\sum_{i=1}^{K} \bar{\lambda}_{i}^{s} C_{\max }^{s}\left(\bar{P}_{i}^{s}\right)$, which is lower than that achieved by the alternative solution due to the following relation:

$$
\begin{aligned}
\bar{C}^{s}=\sum_{i=1}^{K} \bar{\lambda}_{i}^{s} C_{\max }^{s}\left(\bar{P}_{i}^{s}\right)= & \sum_{k \in M} \bar{\lambda}_{k}^{s} C_{\max }^{s}\left(P_{\mathrm{pk}}\right) \\
< & \sum_{k \in M} \bar{\lambda}_{k}^{s} C_{\max }^{s}\left(P_{\mathrm{pk}}\right) \\
& +\hat{\lambda}_{1}^{\mathrm{s}} C_{\max }^{\mathrm{s}}\left(\hat{P}_{1}^{\mathrm{s}}\right) \\
= & \sum_{i=1}^{K} \hat{\lambda}_{i}^{\mathrm{s}} C_{\max }^{\mathrm{s}}\left(\hat{P}_{i}^{\mathrm{s}}\right) \\
= & \hat{C}^{s}
\end{aligned}
$$

where (26) follows from the condition that $\bar{P}_{k}^{s}=P_{\mathrm{pk}}, \forall k \in$ $M$, the inequality in (27) is due to $\hat{\lambda}_{1}^{s}>0$ and $\hat{P}_{1}^{s}>0$, (28) is obtained based on (13)-(18) and (22)-(25), and finally $\hat{C}^{S}$ in (28) denotes the achieved average capacity by the alternative solution. Based on (26)-(29), it is obtained that $\bar{C}^{s}<\hat{C}^{s}$. Therefore, $\left\{\bar{\lambda}_{i}^{\mathrm{p}}, \bar{\lambda}_{i}^{s}, \bar{P}_{i}^{\mathrm{p}}, \bar{P}_{i}^{s}\right\}_{i=1}^{K}$ cannot be optimal and consequently the assumption at the beginning of the proof must be false if $\bar{P}_{k}^{s}=P_{\mathrm{pk}}, \forall k \in M$ for the case that $\bar{\lambda}_{i}^{s}>0, \exists i \in\{1, \ldots, K\}$.

- If $\bar{P}_{k}^{\mathrm{s}}<P_{\mathrm{pk}}, \exists k \in M$, then based on a similar approach to that in Lemma 1 of [30], an alternative solution $\left\{\hat{\lambda}_{i}^{\mathrm{p}}, \hat{\lambda}_{i}^{\mathrm{s}}, \hat{P}_{i}^{\mathrm{p}}\right.$, $\left.\hat{P}_{i}^{s}\right\}_{i=1}^{K}$ can be expressed as

$\hat{\lambda}_{i}^{\mathrm{p}}=\bar{\lambda}_{i}^{\mathrm{p}}, \forall i \in\{1, \ldots, K\}$,

$\hat{P}_{i}^{\mathrm{p}}=\bar{P}_{i}^{\mathrm{p}}, \forall i \in\{1, \ldots, K\}$,

$\hat{P}_{l}^{\mathrm{s}}=\min \left\{P_{\mathrm{pk}}, \bar{P}_{l}^{\mathrm{s}}+\left(P_{\mathrm{av}}-\sum_{i=1}^{K} \bar{\lambda}_{i}^{\mathrm{s}} \bar{P}_{i}^{\mathrm{s}}\right) / \bar{\lambda}_{l}^{\mathrm{s}}\right\}$,

$\hat{P}_{i}^{s}=\bar{P}_{i}^{s}, \forall i \in\{1, \ldots, K\} \backslash\{l\}$,

$\hat{\lambda}_{i}^{s}=\bar{\lambda}_{i}^{s}, \forall i \in\{1, \ldots, K\}$

where $\bar{P}_{l}^{\mathrm{s}}$ is one of the power levels that satisfies $\bar{P}_{l}^{\mathrm{s}}<P_{\mathrm{pk}}$. Since $\hat{P}_{l}^{\mathrm{s}}>\bar{P}_{l}^{\mathrm{s}}$ and $C_{\max }^{\mathrm{s}}(P)$ in $(4)$ is a monotone increasing function of $P$, it is obtained that the alternative solution achieves a larger average capacity than $\left\{\bar{\lambda}_{i}^{\mathrm{p}}, \bar{\lambda}_{i}^{\mathrm{s}}, \bar{P}_{i}^{\mathrm{p}}, \bar{P}_{i}^{\mathrm{s}}\right\}_{i=1}^{K}$ does. Therefore, the assumption at the beginning of the proof must not be true.

Based on the cases specified above, it is concluded by contradiction that the solution of the optimization problem in (3) satisfies the constraint in (3c) with equality; that is, $\sum_{i=1}^{K} \bar{\lambda}_{i}^{\mathrm{p}} \bar{P}_{i}^{\mathrm{p}}+\bar{\lambda}_{i}^{\mathrm{s}} \bar{P}_{i}^{\mathrm{s}}=P_{\mathrm{av}}$.

In the second part of the proof, the aim is to prove that the solution of (3) satisfies the constraint in (3b) with equality. Assume that $\left\{\bar{\lambda}_{i}^{\mathrm{p}}, \bar{\lambda}_{i}^{\mathrm{s}}, \bar{P}_{i}^{\mathrm{p}}, \bar{P}_{i}^{\mathrm{s}}\right\}_{i=1}^{K}$ is the solution of (3) such that $\sum_{i=1}^{K} \bar{\lambda}_{i}^{\mathrm{p}} C_{\mathrm{max}}^{\mathrm{p}}\left(\bar{P}_{i}^{\mathrm{p}}\right)>C_{\text {req. }}$. Since $C_{\text {req }}>0$ by assumption, there exists at least one $\left\{\bar{\lambda}_{i}^{\mathrm{p}}, \bar{P}_{i}^{\mathrm{p}}\right\}$ pair such that $\bar{\lambda}_{i}^{\mathrm{p}}>0$ and $\bar{P}_{i}^{\mathrm{p}}>0$. Let $\left\{\bar{\lambda}_{l}^{\mathrm{p}}, \bar{P}_{l}^{\mathrm{p}}\right\}$ denote one of them. Then, there exists a non-negative $\hat{P}_{l}^{\mathrm{p}}<\bar{P}_{l}^{\mathrm{p}}$ such that $\sum_{i=1}^{K} \hat{\lambda}_{i}^{\mathrm{p}} C_{\max }^{\mathrm{p}}\left(\hat{P}_{i}^{\mathrm{p}}\right) \geq C_{\text {req }}$, where $\hat{\lambda}_{i}^{\mathrm{p}}=\bar{\lambda}_{i}^{\mathrm{p}}$ for all $i \in\{1, \ldots, K\}$ and $\hat{P}_{i}^{\mathrm{p}}=\bar{P}_{i}^{\mathrm{p}}$ for all $i \in\{1, \ldots, K\} \backslash\{l\}$ since $C_{\max }^{\mathrm{p}}(P)$ is a monotone increasing and continuous function of $P$.

- If $\bar{\lambda}_{i}^{s}=0, \forall i \in\{1, \ldots, K\}$, then consider an alternative solution $\left\{\hat{\lambda}_{i}^{\mathrm{p}}, \hat{\lambda}_{i}^{\mathrm{s}}, \hat{P}_{i}^{\mathrm{p}}, \hat{P}_{i}^{\mathrm{s}}\right\}_{i=1}^{K}$, where

$\hat{\lambda}_{1}^{\mathrm{s}}=\bar{\lambda}_{l}^{\mathrm{p}}$, 
$\hat{P}_{1}^{\mathrm{s}}=\bar{P}_{l}^{\mathrm{p}}-\hat{P}_{l}^{\mathrm{p}}$,

$\hat{\lambda}_{i}^{\mathrm{s}}=\bar{\lambda}_{i}^{\mathrm{s}}, \forall i \in\{2, \ldots, K\}$,

$\hat{P}_{i}^{\mathrm{s}}=\bar{P}_{i}^{\mathrm{s}}, \forall i \in\{2, \ldots, K\}$.

- For the case that $\bar{\lambda}_{i}^{s}>0, \exists i \in\{1, \ldots, K\}$, define a set as

$M \triangleq\left\{i \in\{1, \ldots, K\} \mid \bar{\lambda}_{i}^{\mathrm{s}}>0\right\}$.

Next, consider the following cases:

- If $\bar{P}_{k}^{\mathrm{s}}=P_{\mathrm{pk}}, \forall k \in M$, then consider an alternative solution $\left\{\hat{\lambda}_{i}^{\mathrm{p}}, \hat{\lambda}_{i}^{\mathrm{s}}, \hat{P}_{i}^{\mathrm{p}}, \hat{P}_{i}^{\mathrm{s}}\right\}_{i=1}^{K}$, where $\hat{\lambda}_{1}^{\mathrm{s}}$ and $\hat{P}_{1}^{\mathrm{s}}$ are as in (36) and (37), respectively, and

$$
\begin{aligned}
& \hat{\lambda}_{2}^{\mathrm{s}}=\sum_{k \in M} \bar{\lambda}_{k}^{\mathrm{s}}, \\
& \hat{P}_{2}^{\mathrm{s}}=P_{\mathrm{pk}}, \\
& \hat{\lambda}_{i}^{\mathrm{s}}=0, \forall i \in\{3, \ldots, K\}, \\
& \hat{P}_{i}^{\mathrm{s}}=0, \forall i \in\{3, \ldots, K\} .
\end{aligned}
$$

- If $\bar{P}_{k}^{\mathrm{s}}<P_{\mathrm{pk}}, \exists k \in M$, then based on a similar approach to that in Lemma 1 of [30], an alternative solution $\left\{\hat{\lambda}_{i}^{\mathrm{p}}, \hat{\lambda}_{i}^{\mathrm{s}}, \hat{P}_{i}^{\mathrm{p}}\right.$, $\left.\hat{P}_{i}^{s}\right\}_{i=1}^{K}$ can be expressed as

$$
\begin{aligned}
& \hat{P}_{l}^{\mathrm{s}}=\min \left\{P_{\mathrm{pk}}, \bar{P}_{l}^{\mathrm{s}}+\bar{\lambda}_{l}^{\mathrm{p}}\left(\bar{P}_{l}^{\mathrm{p}}-\hat{P}_{l}^{\mathrm{p}}\right) / \bar{\lambda}_{l}^{\mathrm{s}}\right\}, \\
& \hat{P}_{i}^{\mathrm{s}}=\bar{P}_{i}^{\mathrm{s}}, \forall i \in\{1, \ldots, K\} \backslash\{l\}, \\
& \hat{\lambda}_{i}^{\mathrm{s}}=\bar{\lambda}_{i}^{\mathrm{s}}, \forall i \in\{1, \ldots, K\}
\end{aligned}
$$

where $\bar{P}_{l}^{\mathrm{s}}$ is one of the power levels that satisfies $\bar{P}_{l}^{\mathrm{s}}<P_{\mathrm{pk}}$.

Similar to the first part of the proof, all alternative solutions specified for the cases above achieve a larger average capacity than $\left\{\bar{\lambda}_{i}^{\mathrm{p}}, \bar{\lambda}_{i}^{\mathrm{s}}, \bar{P}_{i}^{\mathrm{p}}, \bar{P}_{i}^{s}\right\}_{i=1}^{K}$ does. Therefore, it is proved by contradiction that the solution satisfies the constraint in (3b) with equality; that is, $\sum_{i=1}^{K} \bar{\lambda}_{i}^{\mathrm{p}} C_{\max }^{\mathrm{p}}\left(\bar{P}_{i}^{\mathrm{p}}\right)=C_{\text {req. }}$.

Even though Proposition 2 states that the constraints in (3b) and (3c) are satisfied with equality, it is still difficult to solve the optimization problem in (3). Therefore, the following proposition is presented in order to provide a further simplification for the optimization problem in (3).

Proposition 3. The optimal channel switching strategy based on the optimization problem in (3) employs at most $\min \{3,2 K\}$ communication links.

Proof. If $K \leq 1$, then the assertion in Proposition 3 holds obviously. Otherwise, (if $K>1$ ), then consider the following transformations:

$$
\begin{aligned}
v_{i} & =\left\{\begin{array}{ll}
\lambda_{i}^{\mathrm{p}}, & \text { if } i \leq K \\
\lambda_{m}^{\mathrm{s}}, & \text { if } i>K
\end{array}, \forall i \in\{1, \ldots, 2 K\}\right. \\
P_{i} & =\left\{\begin{array}{ll}
P_{i}^{\mathrm{p}}, & \text { if } i \leq K \\
P_{m}^{\mathrm{s}}, & \text { if } i>K
\end{array}, \forall i \in\{1, \ldots, 2 K\}\right.
\end{aligned}
$$

where $m \triangleq i-K$. Also, define the following functions:

$$
\begin{aligned}
& C_{\max , i}^{\mathrm{p}}(P)=\left\{\begin{array}{ll}
C_{\max }^{\mathrm{p}}(P), & \text { if } i \leq K \\
0, & \text { if } i>K
\end{array}, \forall i \in\{1, \ldots, 2 K\}\right. \\
& C_{\max , i}^{\mathrm{s}}(P)=\left\{\begin{array}{ll}
0, & \text { if } i \leq K \\
C_{\max }^{\mathrm{s}}(P), & \text { if } i>K
\end{array}, \forall i \in\{1, \ldots, 2 K\}\right.
\end{aligned}
$$

for all $P \in\left[0, P_{\mathrm{pk}}\right]$. Based on the transformations in (47) and (48) and the functions in (49) and (50), the optimization problem in (3) can be written in the following form:

$$
\begin{aligned}
\max _{\left\{v_{i}, P_{i}\right\}_{i=1}^{2 K}} & \sum_{i=1}^{2 K} v_{i} C_{\max , i}^{\mathrm{s}}\left(P_{i}\right) \\
\text { subject to } & \sum_{i=1}^{2 K} v_{i} C_{\max , i}^{\mathrm{p}}\left(P_{i}\right) \geq C_{\mathrm{req}} \\
& \sum_{i=1}^{2 K} v_{i} P_{i} \leq P_{\mathrm{av}}, P_{i} \in\left[0, P_{\mathrm{pk}}\right], \forall i \in\{1, \ldots, 2 K\} \\
& \sum_{i=1}^{2 K} v_{i}=1, v_{i} \in[0,1], \forall i \in\{1, \ldots, 2 K\}
\end{aligned}
$$

Next, define the following sets:

$$
\begin{aligned}
& \mathcal{V}=\left\{\left(\sum_{i=1}^{2 K} v_{i} C_{\max , i}^{\mathrm{p}}\left(P_{i}\right), \sum_{i=1}^{2 K} v_{i} C_{\max , i}^{\mathrm{s}}\left(P_{i}\right), \sum_{i=1}^{2 K} v_{i} P_{i}\right) \in \mathbb{R}^{3}\right. \\
& \left.\mid \sum_{i=1}^{2 K} v_{i}=1, v_{i} \in[0,1], P_{i} \in\left[0, P_{\mathrm{pk}}\right], \forall i \in\{1, \ldots, 2 K\}\right\}
\end{aligned}
$$

$\mathcal{W}=\left\{w=\left\{u_{1}, \ldots, u_{2 K}\right\} \mid u_{i} \in \mathcal{U}_{i}, \forall i \in\{1, \ldots, 2 K\}\right\}$

where

$\begin{aligned} \mathcal{U}_{i}= & \left\{\left(P, C_{\max , i}^{\mathrm{p}}(P), C_{\max , i}^{\mathrm{s}}(P)\right) \in \mathbb{R}^{3} \mid P \in\left[0, P_{\mathrm{pk}}\right]\right\}, \\ & \forall i \in\{1, \ldots, 2 K\} .\end{aligned}$

It is noted that set $\mathcal{V}$ includes the solution of the optimization problem in (51) by definition. Let $\mathcal{W}_{i}$ represent the $i$ th element of set $\mathcal{W}$, which is also a set. Then, set $\mathcal{V}$ is equal to the union of the convex hulls of set $\mathcal{W}_{i}, \forall i \in\{1, \ldots,|\mathcal{W}|\}$; that is, $\mathcal{V}=$ $\bigcup_{i=1}^{|\mathcal{W}|} \operatorname{conv}\left(\mathcal{W}_{i}\right)$. Therefore, $\bigcup_{i=1}^{|\mathcal{W}|} \operatorname{conv}\left(\mathcal{W}_{i}\right)$ also includes the solution of the optimization problem in (51). The definition of union implies that the solution of (51) is an element of $\operatorname{conv}\left(\mathcal{W}_{i}\right)$ for some $i \in\{1, \ldots,|\mathcal{W}|\}$. Without loss of generality, let $l$ be one of them. Since the optimization problem in (51) is a maximization problem, the solution of (51) resides on the boundary of the convex hull of set $\mathcal{W}_{l}$. Then, by Carathéodory's theorem [36,37], any point on the boundary of the convex hull of set $\mathcal{W}_{l}$ can be represented by a convex combination of at most $d$ points in set $\mathcal{W}_{l}$, where $d$ is the dimension of space in which $\mathcal{W}_{l}$ resides. Since $\mathcal{W}_{l} \subset \mathbb{R}^{3}$ and the optimal solution of (51) corresponds to a point on the boundary of $\operatorname{conv}\left(\mathcal{W}_{l}\right)$, the optimal channel switching strategy employs at most 3 communication links.

Based on Proposition 3 and the study in [30], the optimal channel switching strategy can be investigated as follows: Let $\bar{C}_{\text {req }}$ denote the achieved maximum average capacity for the communication between the transmitter and the primary receiver when there is no secondary receiver in the system. Then, $\bar{C}_{\text {req }}$ can be calculated as follows:

$$
\begin{array}{r}
\bar{C}_{\text {req }}=\max _{\left\{\lambda_{i}^{\mathrm{p}}, P_{i}^{\mathrm{p}}\right\}_{i=1}^{K}} \sum_{i=1}^{K} \lambda_{i}^{\mathrm{p}} C_{\mathrm{max}}^{\mathrm{p}}\left(P_{i}^{\mathrm{p}}\right) \\
\text { subject to } \sum_{i=1}^{K} \lambda_{i}^{\mathrm{p}} P_{i}^{\mathrm{p}}=P_{\mathrm{av}},
\end{array}
$$




$$
\begin{aligned}
& P_{i}^{\mathrm{p}} \in\left[0, P_{\mathrm{pk}}\right], \forall i \in\{1, \ldots, K\} \\
& \sum_{i=1}^{K} \lambda_{i}^{\mathrm{p}}=1, \lambda_{i}^{\mathrm{p}} \in[0,1], \forall i \in\{1, \ldots, K\}
\end{aligned}
$$

If the maximum average capacity achieved by the optimization problem in (55) is strictly lower than the minimum average capacity requirement for the primary receiver (i.e., $\bar{C}_{\text {req }}<C_{\text {req }}$ ), then there is no possible channel switching strategy for the problem in (2) since the optimization problem in (3) is infeasible. If $\bar{C}_{\text {req }}=C_{\text {req }}$, the optimal channel switching strategy corresponds to switching between at most two channels between the transmitter and the primary receiver based on the optimization problem in (2) and Proposition 4 in [30]. In that case, no communication is performed between the transmitter and the secondary receiver. Therefore, the achieved maximum average capacity is $C^{\star}=0$. Finally, if $\bar{C}_{\text {req }}>C_{\text {req }}$, then the optimal channel switching strategy corresponds to one of the following two strategies:

- Strategy-1 (Communicate with the primary receiver over at most two channels and employ single channel for the secondary receiver): In this strategy, the transmitter employs one or two channels to satisfy the minimum average capacity requirement of the primary receiver and uses only one channel in order to maximize the average capacity of the communication to the secondary receiver. The achieved maximum average capacity for the communication to the secondary receiver, de-

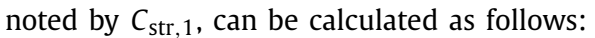

$$
\begin{aligned}
C_{\mathrm{str}, 1}= & \max _{\lambda_{1}, \lambda_{2}, \lambda_{3}, P_{1}, P_{2}, P_{3}} \lambda_{1} C_{\max }^{\mathrm{s}}\left(P_{1}\right) \\
\text { subject to } & \lambda_{2} C_{\max }^{\mathrm{p}}\left(P_{2}\right)+\lambda_{3} C_{\max }^{\mathrm{p}}\left(P_{3}\right)=C_{\mathrm{req}} \\
& \lambda_{1} P_{1}+\lambda_{2} P_{2}+\lambda_{3} P_{3}=P_{\mathrm{av}}, \\
& P_{1}, P_{2}, P_{3} \in\left[0, P_{\mathrm{pk}}\right], \\
& \lambda_{1}+\lambda_{2}+\lambda_{3}=1, \lambda_{1}, \lambda_{2}, \lambda_{3} \in[0,1]
\end{aligned}
$$

\section{- Strategy-2 (Communicate with the secondary receiver over at} most two channels and employ single channel for the primary receiver): In this case, the transmitter maximizes the average capacity of the communication to the secondary receiver by employing one or two channels while meeting the minimum average capacity requirement for the primary receiver via communication over a single channel. In this case, the achieved average capacity can be expressed as

$$
\begin{aligned}
C_{\mathrm{str}, 2}= & \max _{\lambda_{1}, \lambda_{2}, \lambda_{3}, P_{1}, P_{2}, P_{3}} \lambda_{1} C_{\max }^{\mathrm{s}}\left(P_{1}\right)+\lambda_{2} C_{\max }^{\mathrm{s}}\left(P_{2}\right) \\
\text { subject to } & \lambda_{3} C_{\max }^{\mathrm{p}}\left(P_{3}\right)=C_{\mathrm{req}} \\
& \lambda_{1} P_{1}+\lambda_{2} P_{2}+\lambda_{3} P_{3}=P_{\mathrm{av}}, \\
& P_{1}, P_{2}, P_{3} \in\left[0, P_{\mathrm{pk}}\right] \\
& \lambda_{1}+\lambda_{2}+\lambda_{3}=1, \lambda_{1}, \lambda_{2}, \lambda_{3} \in[0,1]
\end{aligned}
$$

Based on Strategy 1 and Strategy 2, the maximum average capacity for the communication between the transmitter and the secondary receiver, which is the solution of (2), can be calculated as

$C^{\star}=\max \left\{C_{\mathrm{str}, 1}, C_{\mathrm{str}, 2}\right\}$

where $C_{\mathrm{str}, 1}$ and $C_{\mathrm{str}, 2}$ are as in (56) and (57), respectively.

It is important to note that the optimization problems in (56) and (57) have significantly lower computational complexity compared to the original optimization problem in (2) since each of
(56) and (57) requires a search only over a 3 dimensional space ${ }^{5}$ whereas a search over a $4 K$ dimensional space is required for the problem in (2), where $K>1$.

\section{Optimal channel switching in the presence of multiple primary receivers}

In the presence of multiple primary receivers, each having an individual minimum average capacity requirement, the optimization problem in (2) can be extended as follows:

$$
\begin{aligned}
& \max _{\left\{\lambda_{i}^{\mathrm{s}}, P_{i}^{\mathrm{s}},\left\{\lambda_{i}^{\mathrm{p}_{j}}, P_{i}^{\mathrm{p}_{j}}\right\}_{j=1}^{N}\right\}_{i=1}^{K}} \sum_{i=1}^{K} \lambda_{i}^{\mathrm{s}} C_{i}^{\mathrm{s}}\left(P_{i}^{\mathrm{s}}\right) \\
\text { subject to } & \sum_{i=1}^{K} \lambda_{i}^{\mathrm{p}_{j}} C_{i}^{\mathrm{p}_{j}}\left(P_{i}^{\mathrm{p}_{j}}\right) \geq C_{\mathrm{req}}^{j}, \forall j \in\{1, \ldots, N\}, \\
& \sum_{i=1}^{K}\left(\lambda_{i}^{\mathrm{s}} P_{i}^{\mathrm{s}}+\sum_{j=1}^{N} \lambda_{i}^{\mathrm{p}_{j}} P_{i}^{\mathrm{p}_{j}}\right) \leq P_{\mathrm{av}}, \\
& P_{i}^{\mathrm{s}}, P_{i}^{\mathrm{p}_{j}} \in\left[0, P_{\mathrm{pk}}\right], \forall i \in\{1, \ldots, K\}, \forall j \in\{1, \ldots, N\}, \\
& \sum_{i=1}^{K}\left(\lambda_{i}^{\mathrm{s}}+\sum_{j=1}^{N} \lambda_{i}^{\mathrm{p}_{j}}\right)=1, \\
& \lambda_{i}^{\mathrm{s}}, \lambda_{i}^{\mathrm{p}_{j}} \in[0,1], \forall i \in\{1, \ldots, K\}, \forall j \in\{1, \ldots, N\},
\end{aligned}
$$

where $\lambda_{i}^{\mathrm{p}_{j}}$ and $P_{i}^{\mathrm{p}_{j}}$ denote, respectively, the time-sharing factor and the average transmit power allocated to channel $i$ for the communication between the transmitter and the $j$ th primary receiver, $N$ is the number of primary receivers in the system, $C_{i}^{\mathrm{p}_{j}}(P)$ as defined in (1), $C_{\text {req }}^{j}$ is the minimum average capacity requirement for the $j$ th primary receiver, and the other parameters are as in (2).

It is noted that the optimization problem in (2) is a special case of (59) when there exists only one primary receiver; that is, when $N=1$. Therefore, it is in general more difficult to solve the optimization problem in (59) since it requires a search over a $2 K(N+1)$ dimensional space, which is higher than $4 K$, corresponding to (2), for $N>1$. However, the results obtained for the problem in (2) can be extended for (59), as explained in the following remark.

Remark 1. Based on a similar approach to that in Proposition 1, it can be shown that an alternative optimization problem to the problem in (59) can be obtained. Also, the approach in Proposition 2 also holds for the optimization problem in (59) and consequently the solution of (59) satisfies the constraints in (59b) and (59c) with equality. Moreover, similar to the proof in Proposition 3, it can be stated for the optimization problem in (59) that the optimal channel switching strategy based on (59) employs at most $\min \{N+2, K(N+1)\}$ communication links in the system, where $K(N+1)$ links are available in total. Specifically, the optimal channel switching strategy can be realized by switching among at most $(N+2)$ communication links in the presence of multiple available channels in the system; that is, $K>1$.

It is concluded from Remark 1 that the solution of (59) can be calculated by solving a total of $(N+1)$ optimization problems,

\footnotetext{
5 Note that the search space dimensions of the optimization problems in (56) and (57) are obtained by substituting the equality constraints in (56b)-(56d) and (57b)-(57d) into the objective functions in (56a) and (57a), respectively.
} 


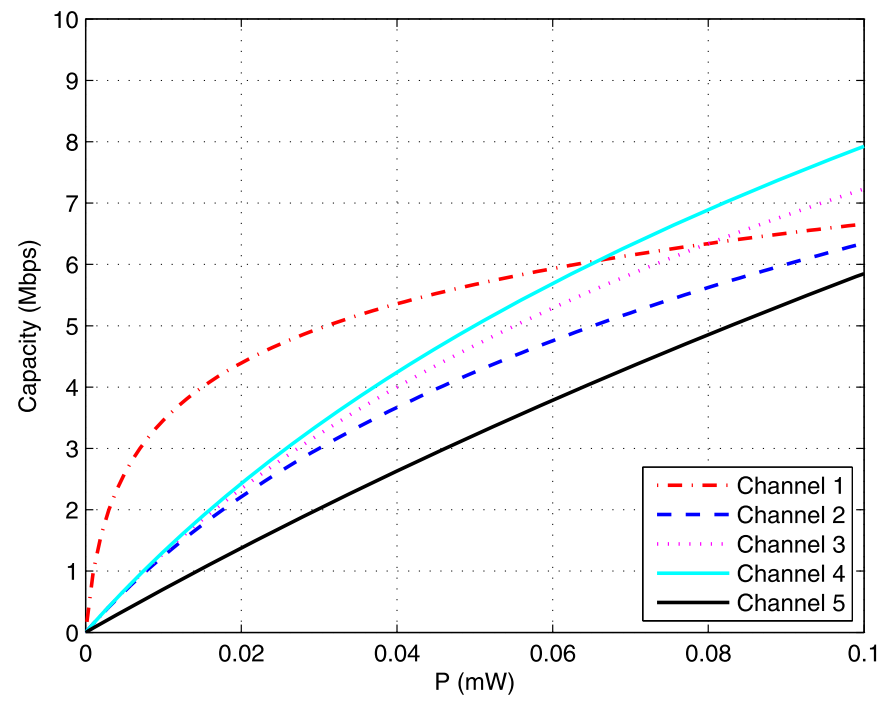

Fig. 2. Capacity of each link versus power for the communication between the transmitter and the primary receiver, where $B_{1}=1 \mathrm{MHz}, B_{2}=3 \mathrm{MHz}, B_{3}=4 \mathrm{MHz}$, $B_{4}=5 \mathrm{MHz}, B_{5}=10 \mathrm{MHz}, N_{1}=N_{2}=N_{3}=N_{4}=N_{5}=10^{-12} \mathrm{~W} / \mathrm{Hz},\left|h_{1}^{\mathrm{p}}\right|^{2}=1$, $\left|h_{2}^{\mathrm{p}}\right|^{2}=0.1,\left|h_{3}^{\mathrm{p}}\right|^{2}=0.1,\left|h_{4}^{\mathrm{p}}\right|^{2}=0.1$, and $\left|h_{5}^{\mathrm{p}}\right|^{2}=0.05$.

each requiring a search over a $2(N+2)$ dimensional space, and then choosing the maximum among the obtained average capacities. Hence, the optimal channel switching strategy based on the optimization problem in (59) can be obtained with low computational complexity.

For complexity comparisons, assume that there exist finitely many possible values of $\lambda_{i}^{k}$ and $P_{i}^{k}$ for each $k \in\{\mathrm{p}, \mathrm{s}\}$ and $i \in$ $\{1, \ldots, K\}$, where $\lambda_{i}^{k} \in[0,1]$ and $P_{i}^{k} \in\left[0, P_{\mathrm{pk}}\right]$ for all $k \in\{\mathrm{p}, \mathrm{s}\}$ and $i \in\{1, \ldots, K\}$. Let $N_{\lambda}$ denote the number of different $\lambda$ values for $\lambda \in[0,1]$ and $N_{P}$ represent the number of different $P$ values for $P \in\left[0, P_{\mathrm{pk}}\right]$. Then, the original optimization problem in (2) has a computational complexity of $\mathcal{O}\left(N_{\lambda}^{2 K} \times N_{P}^{2 K}\right)$. On the other hand, the complexity of each optimization problem in (56) and (57) is in the order of $\mathcal{O}\left(N_{\lambda}^{3} \times N_{P}^{3}\right)$. Therefore, in the presence of multiple available channels, instead of solving the original optimization problem in (2) with a complexity of $\mathcal{O}\left(N_{\lambda}^{2 K} \times N_{P}^{2 K}\right)$ where $K>1$, the solution of (2) can be obtained with a lower computational complexity by solving two optimization problems in (56) and (57), each having a computational complexity of $\mathcal{O}\left(N_{\lambda}^{3} \times N_{P}^{3}\right)$. In the presence of $N$ primary receivers in the communication system, the complexity of the optimization problem in (59) is $\mathcal{O}\left(N_{\lambda}^{K(N+1)} \times N_{P}^{K(N+1)}\right)$. However, the solution of (59) can be calculated with a lower complexity by solving $N+1$ optimization problems, each having a computational complexity of $\mathcal{O}\left(N_{\lambda}^{N+2} \times N_{P}^{N+2}\right)$.

\section{Numerical results}

In this section, several numerical examples are presented to investigate the performance of the proposed strategies and to illustrate the optimal strategy for various values of the average power limit and the minimum average capacity requirement for the primary receiver. To that aim, a communication system is considered with $K=5$ channels, the bandwidths and the noise levels of which are given by $B_{1}=1 \mathrm{MHz}, B_{2}=3 \mathrm{MHz}, B_{3}=4 \mathrm{MHz}, B_{4}=$ $5 \mathrm{MHz}, B_{5}=10 \mathrm{MHz}$, and $N_{1}=N_{2}=N_{3}=N_{4}=N_{5}=10^{-12} \mathrm{~W} / \mathrm{Hz}$ (cf. (1)). It is assumed that all the channels are available for the transmitter and can be used to communicate with both the primary and secondary receivers. Also, for these channels, the channel power gains from the transmitter to the primary and secondary receivers are given by $\left|h_{1}^{\mathrm{p}}\right|^{2}=1,\left|h_{2}^{\mathrm{p}}\right|^{2}=0.1,\left|h_{3}^{\mathrm{p}}\right|^{2}=0.1,\left|h_{4}^{\mathrm{p}}\right|^{2}=0.1$, $\left|h_{5}^{\mathrm{p}}\right|^{2}=0.05,\left|h_{1}^{\mathrm{s}}\right|^{2}=1,\left|h_{2}^{\mathrm{s}}\right|^{2}=0.1,\left|h_{3}^{\mathrm{s}}\right|^{2}=0.1,\left|h_{4}^{\mathrm{s}}\right|^{2}=0.1$, and

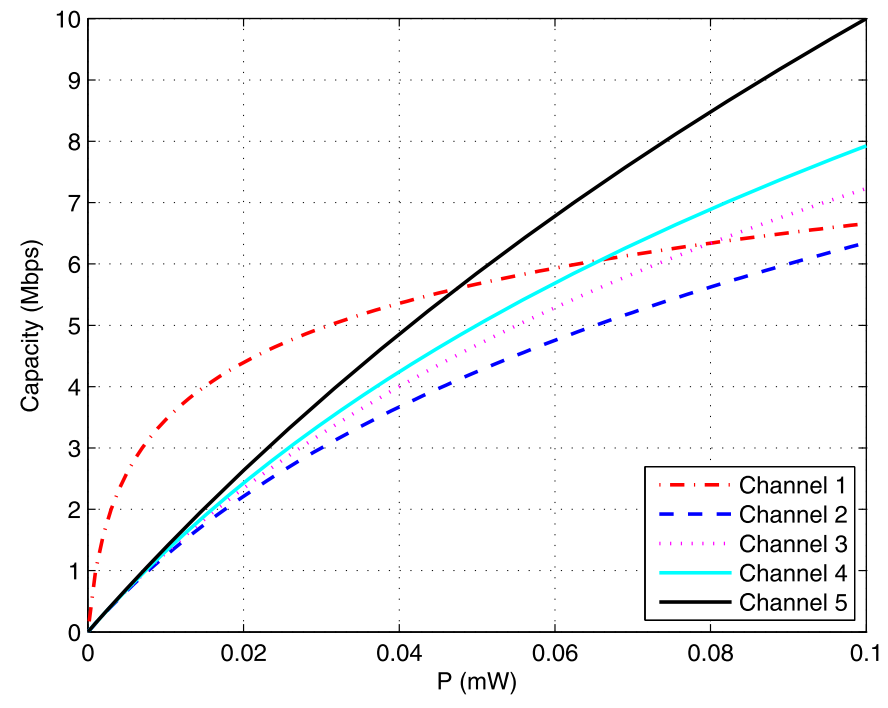

Fig. 3. Capacity of each link versus power for the communication between the transmitter and the secondary receiver, where $B_{1}=1 \mathrm{MHz}, B_{2}=3 \mathrm{MHz}, B_{3}=4 \mathrm{MHz}$, $B_{4}=5 \mathrm{MHz}, B_{5}=10 \mathrm{MHz}, N_{1}=N_{2}=N_{3}=N_{4}=N_{5}=10^{-12} \mathrm{~W} / \mathrm{Hz},\left|h_{1}^{\mathrm{s}}\right|^{2}=1$, $\left|h_{2}^{\mathrm{s}}\right|^{2}=0.1,\left|h_{3}^{\mathrm{s}}\right|^{2}=0.1,\left|h_{4}^{\mathrm{s}}\right|^{2}=0.1$, and $\left|h_{5}^{\mathrm{s}}\right|^{2}=0.1$.

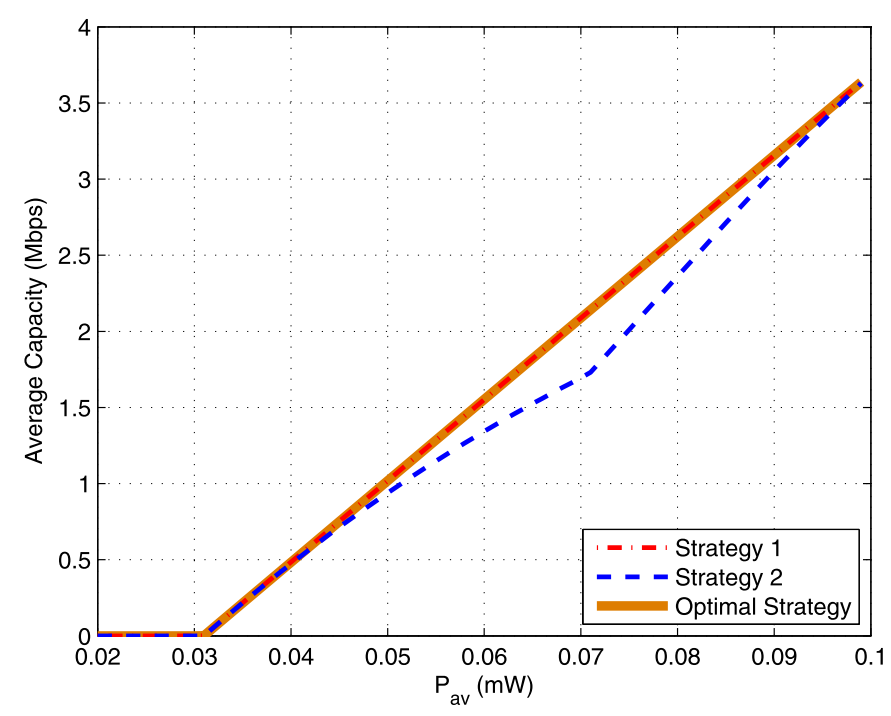

Fig. 4. Average capacity versus average power limit for Strategy 1, Strategy 2, and the optimal channel switching strategy for the scenario in Fig. 2 and Fig. 3, where $C_{\text {req }}=5$ Mbps.

$\left|h_{5}^{\mathrm{s}}\right|^{2}=0.1$. In this scenario, the peak power constraint in (2) is set to $P_{\mathrm{pk}}=0.1 \mathrm{~mW}$. The capacity of each link available for the transmitter to communicate with the primary and secondary receivers is plotted as a function of power in Fig. 2 and Fig. 3, respectively.

In order to investigate the effect of the average power limit on the performance of the optimal channel switching strategies, the minimum average capacity constraint for the primary receiver in (2) is set to $C_{\text {req }}=5$ Mbps first. Then, by considering the channel links in Fig. 2 and Fig. 3, the optimal average capacities are obtained for different average power limits $\left(P_{\mathrm{av}}\right)$ based on Strategy 1 in (56) and Strategy 2 in (57), and the achieved maximum average capacities are presented in Fig. 4. From Fig. 4, it is observed that $C^{\star}=0$ for $P_{\mathrm{av}}<0.031 \mathrm{~mW}$ since there is no feasible solution of the optimization problem in (2) for $C_{\text {req }}=5 \mathrm{Mbps}$ and $P_{\mathrm{av}}<0.031 \mathrm{~mW}$. On the other hand, for $P_{\mathrm{av}} \geq 0.031 \mathrm{~mW}$, the optimal channel switching strategy can be obtained based on (56) and (57), and it corresponds to Strategy 1 for all $P_{\mathrm{av}} \geq 0.031 \mathrm{~mW}$ since Strategy 1 outperforms Strategy 2 in terms of the achiev- 
able maximum average capacity for the communication to the secondary receiver. Therefore, the optimal strategy for the transmitter is to communicate with the primary receiver over at most two channels and to employ a single channel for the secondary receiver. It is also noted that the solutions of the optimization problem in (2) for different values of $P_{\mathrm{av}} \geq 0.031 \mathrm{~mW}$ satisfy the average power and minimum average capacity requirement constraints with equality as Proposition 2 states.

To analyze the optimal strategy in Fig. 4 in more detail, Table 1 presents the solutions of the optimal strategy for various values of the average power limit, $P_{\mathrm{av}}$. In the table, the optimal solution is represented by parameters $\lambda_{1}^{k}, \lambda_{2}^{k}, P_{1}^{k}, P_{2}^{k}, i^{k}$, and $j^{k}$ for all $k \in\{\mathrm{p}, \mathrm{s}\}$, meaning that channel $i^{k}$ is used with time-sharing factor $\lambda_{1}^{k}$ and power $P_{1}^{k}$, and channel $j^{k}$ is employed with timesharing factor $\lambda_{2}^{k}$ and power $P_{2}^{k}$ to communicate with the primary receiver for $k=\mathrm{p}$ and with the secondary receiver for $k=\mathrm{s}$. It is deduced from Table 1 that there is no possible channel switching strategy for $P_{\mathrm{av}}=0.01 \mathrm{~mW}, P_{\mathrm{av}}=0.02 \mathrm{~mW}$, and $P_{\mathrm{av}}=0.03 \mathrm{~mW}$. On the other hand, for the other $P_{\mathrm{av}}$ values in Table 1, the optimal strategy for the average capacity maximization of the secondary receiver is to communicate with the primary receiver over channel 1 and channel 4 via channel switching, and to employ channel 5 exclusively to communicate with the secondary receiver.

In Fig. 5, the maximum average capacities for the strategies stated in Fig. 4 are plotted versus the minimum average capacity requirement, $C_{\text {req }}$, based on the scenario in Fig. 2 and Fig. 3. The average power limit in (2) is set to $P_{\mathrm{av}}=0.05 \mathrm{~mW}$. From Fig. 5, it is obtained that Strategy 2 is the optimal strategy for $C_{\text {req }} \in(0,2.6]$ Mbps whereas Strategy 1 is optimal for $C_{\text {req }} \in$ $[3.9,5.8] \mathrm{Mbps}$. On the other hand, for $C_{\text {req }} \in(2.6,3.9) \mathrm{Mbps}$, both Strategy 1 and Strategy 2 are optimal since the communication is performed over a single channel for each of primary and secondary receivers. Also, it is noted that there is no optimal strategy for $C_{\text {req }}>5.8 \mathrm{Mbps}$ since $\bar{C}_{\text {req }}$ in (55) cannot achieve a capacity equal to or higher than $C_{\text {req }}$; that is, $\bar{C}_{\text {req }}<C_{\text {req }}$.

Similar to Table 1 , the solutions of the optimal strategies for various values of the minimum average capacity requirement of the primary receiver, $C_{\text {req, }}$, are presented in Table 2 . It is noted from Table 2 that the optimal strategy for the values satisfying $C_{\text {req }} \leq 2.5$ Mbps corresponds to the exclusive use of channel 1 for the primary receiver and to channel switching between channel 1 and channel 5 for the secondary receiver whereas for the values of $C_{\text {req }}$ with $C_{\text {req }} \geq 4.0 \mathrm{Mbps}$ and $C_{\text {req }} \leq 5.5 \mathrm{Mbps}$, it corresponds to switching between channel 1 and channel 4 for the primary receiver and to the use of channel 5 only for the secondary receiver. Also, for $C_{\text {req }}=3.0 \mathrm{Mbps}$ and $C_{\text {req }}=3.5 \mathrm{Mbps}$, the optimal strategy is to employ channel 1 and channel 5 for the primary and secondary receivers, respectively. In this case, it is observed that

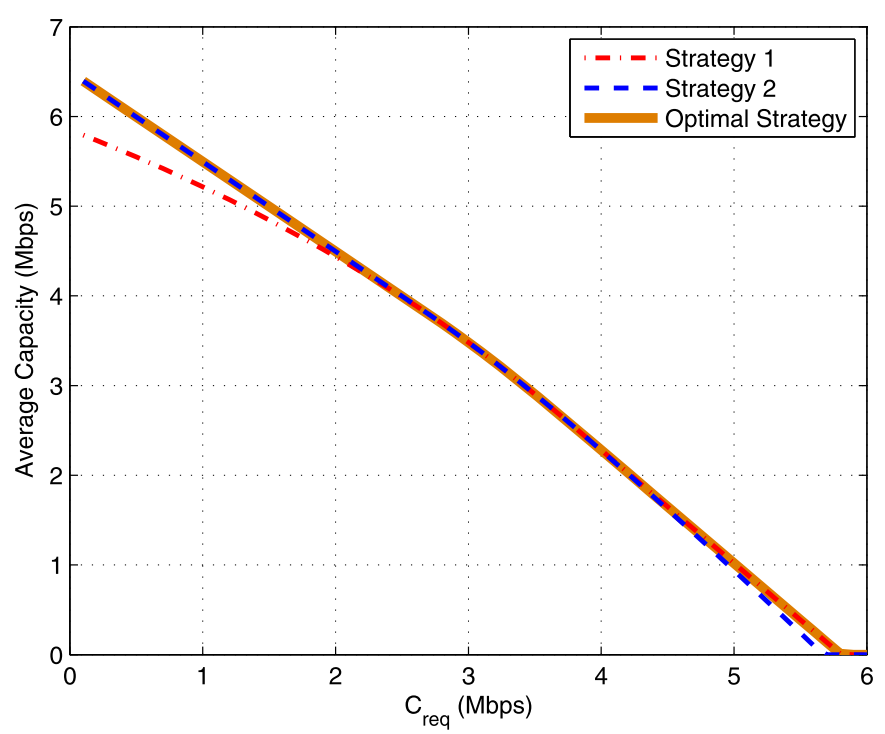

Fig. 5. Average capacity versus minimum average capacity requirement for Strategy 1 , Strategy 2, and the optimal channel switching strategy for the scenario in Fig. 2 and Fig. 3, where $P_{\mathrm{av}}=0.05 \mathrm{~mW}$.

Table 1

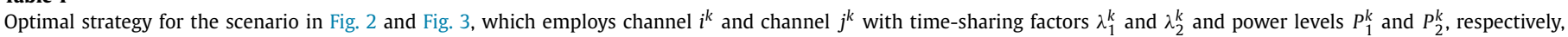
to communicate with the primary receiver $(k=\mathrm{p})$ and the secondary receiver $(k=\mathrm{s})$.

\begin{tabular}{|c|c|c|c|c|c|c|c|c|c|c|c|c|}
\hline$P_{\mathrm{av}}(\mathrm{mW})$ & $\lambda_{1}^{\mathrm{p}}$ & $P_{1}^{\mathrm{p}}$ & $i^{\mathrm{p}}$ & $\lambda_{2}^{p}$ & $P_{2}^{\mathrm{p}}$ & $j^{\mathrm{P}}$ & $\lambda_{1}^{\mathrm{s}}$ & $P_{1}^{\mathrm{s}}$ & $i^{\mathrm{s}}$ & $\lambda_{2}^{\mathrm{s}}$ & $P_{2}^{\mathrm{s}}$ & $j^{\mathrm{s}}$ \\
\hline 0.01 & - & - & - & - & - & - & - & - & - & - & - & - \\
\hline 0.02 & - & - & - & - & - & - & - & - & - & - & - & - \\
\hline 0.03 & - & - & - & - & - & - & - & - & - & - & - & - \\
\hline 0.04 & 0.8963 & 0.0331 & 1 & 0.0552 & 0.1 & 4 & 0.0484 & 0.1 & 5 & - & - & - \\
\hline 0.05 & 0.7469 & 0.0331 & 1 & 0.1512 & 0.1 & 4 & 0.1019 & 0.1 & 5 & - & - & - \\
\hline 0.06 & 0.5975 & 0.0331 & 1 & 0.2471 & 0.1 & 4 & 0.1553 & 0.1 & 5 & - & - & - \\
\hline 0.07 & 0.4482 & 0.0331 & 1 & 0.3431 & 0.1 & 4 & 0.2088 & 0.1 & 5 & - & - & - \\
\hline 0.08 & 0.2988 & 0.0331 & 1 & 0.439 & 0.1 & 4 & 0.2622 & 0.1 & 5 & - & - & - \\
\hline 0.09 & 0.1494 & 0.0331 & 1 & 0.535 & 0.1 & 4 & 0.3156 & 0.1 & 5 & - & - & - \\
\hline
\end{tabular}

Table 2

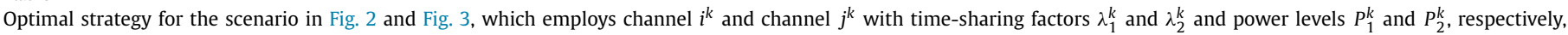
to communicate with the primary receiver $(k=\mathrm{p})$ and the secondary receiver $(k=\mathrm{s})$.

\begin{tabular}{|c|c|c|c|c|c|c|c|c|c|c|c|c|}
\hline$C_{\text {req }}(\mathrm{Mbps})$ & $\lambda_{1}^{\mathrm{p}}$ & $P_{1}^{\mathrm{p}}$ & $i^{\mathrm{P}}$ & $\lambda_{2}^{\mathrm{p}}$ & $P_{2}^{\mathrm{p}}$ & $j^{\mathrm{P}}$ & $\lambda_{1}^{s}$ & $P_{1}^{\mathrm{s}}$ & $i^{\mathrm{s}}$ & $\lambda_{2}^{\mathrm{s}}$ & $P_{2}^{\mathrm{s}}$ & $j^{\mathrm{s}}$ \\
\hline 0.5 & 0.1146 & 0.0196 & 1 & - & - & - & 0.5071 & 0.0196 & 1 & 0.3783 & 0.1 & 5 \\
\hline 1 & 0.2292 & 0.0196 & 1 & - & - & - & 0.3925 & 0.0196 & 1 & 0.3783 & 0.1 & 5 \\
\hline 1.5 & 0.3438 & 0.0196 & 1 & - & - & - & 0.2779 & 0.0196 & 1 & 0.3783 & 0.1 & 5 \\
\hline 2 & 0.4584 & 0.0196 & 1 & - & - & - & 0.1633 & 0.0196 & 1 & 0.3783 & 0.1 & 5 \\
\hline 2.5 & 0.573 & 0.0196 & 1 & - & - & - & 0.0487 & 0.0196 & 1 & 0.3783 & 0.1 & 5 \\
\hline 3 & 0.6518 & 0.0233 & 1 & - & - & - & 0.3482 & 0.1 & 5 & - & - & - \\
\hline 3.5 & 0.7096 & 0.0295 & 1 & - & - & - & 0.2904 & 0.1 & 5 & - & - & - \\
\hline 4 & 0.7469 & 0.0331 & 1 & 0.025 & 0.1 & 4 & 0.2281 & 0.1 & 5 & - & - & - \\
\hline 4.5 & 0.7469 & 0.0331 & 1 & 0.0881 & 0.1 & 4 & 0.165 & 0.1 & 5 & - & - & - \\
\hline 5 & 0.7469 & 0.0331 & 1 & 0.1512 & 0.1 & 4 & 0.1019 & 0.1 & 5 & - & - & - \\
\hline 5.5 & 0.7469 & 0.0331 & 1 & 0.2143 & 0.1 & 4 & 0.0388 & 0.1 & 5 & - & - & - \\
\hline 6 & - & - & - & - & - & - & - & - & - & - & - & - \\
\hline
\end{tabular}


both Strategy 1 and Strategy 2 are optimal. Lastly, there is no optimal channel switching strategy for $C_{\text {req }}=6.0 \mathrm{Mbps}$.

\section{Concluding remarks}

In this study, the optimal channel switching problem has been investigated for average capacity maximization in the presence of multiple receivers in a communication system where multiple AWGN channels are available for a transmitter to communicate with the receivers. First, the optimal channel switching problem has been presented for the communication of a transmitter with the primary and secondary receivers in the presence of the minimum average capacity requirement of the primary receiver and the average and peak power constraints. Then, an equivalent optimization problem has been proposed and it has been proved that the solution of this problem satisfies the constraints in equality. Based on the proposed optimization problems, it has been shown that the optimal channel switching strategy does not involve more than three communication links when multiple channels are available in the communication system. Furthermore, the possible optimal channel switching scenarios have been specified in terms of the number of channels required for the transmitter to communicate with the primary and secondary receivers in order to achieve the maximum average capacity of the communication to the secondary receiver while fulfilling the minimum average capacity requirement of the primary receiver. Numerical examples have been provided to illustrate the theoretical results and to demonstrate the benefits of channel switching.

\section{References}

[1] A.J. Goldsmith, P.P. Varaiya, Capacity of fading channels with channel side information, IEEE Trans. Inf. Theory 43 (6) (Nov. 1997) 1986-1992.

[2] X. Kang, Y.C. Liang, A. Nallanathan, H.K. Garg, R. Zhang, Optimal power allocation for fading channels in cognitive radio networks: ergodic capacity and outage capacity, IEEE Trans. Wirel. Commun. 8 (2) (Feb. 2009) 940-950.

[3] F. Zhou, N.C. Beaulieu, Z. Li, J. Si, P. Qi, Energy-efficient optimal power allocation for fading cognitive radio channels: ergodic capacity, outage capacity, and minimum-rate capacity, IEEE Trans. Wirel. Commun. 15 (4) (Apr. 2016) 2741-2755.

[4] D. Xu, Z. Feng, P. Zhang, Minimum average BER power allocation for fading channels in cognitive radio networks, in: IEEE Wireless Commun. and Networking Conf. (WCNC), Mar. 2011, pp. 78-83.

[5] P.H. Reddy, V.N. Kumar, M.Z.A. Khan, M.B. Srinivas, Optimal power allocation in space and time in MISO Rayleigh fading channels with peak to average power ratio constraint, in: IEEE Sarnoff Symposium, Apr. 2007, pp. 1-6.

[6] L. Goldfeld, V. Lyandres, D. Wulich, Minimum BER power loading for OFDM in fading channel, IEEE Trans. Commun. 50 (11) (Nov. 2002) 1729-1733.

[7] A.P. Iserte, M.A.L. Hernandez, A.I. Perez-Neira, Robust power allocation for minimum BER in a SISO-OFDM system, in: IEEE Int. Conf. on Commun. (ICC), vol. 2, May 2003, pp. 1263-1267.

[8] P. Zhang, H.C. Yang, Minimum-BER power allocation for multicarrier systems with outdated channel state information, in: 2007 IEEE Canadian Conference on Electrical and Computer Engineering (CCECE), Apr. 2007, pp. 180-183.

[9] C. Huang, R. Zhang, S. Cui, Optimal power allocation for outage probability minimization in fading channels with energy harvesting constraints, IEEE Trans. Wirel. Commun. 13 (2) (Feb. 2014) 1074-1087.

[10] L. Li, A. Goldsmith, Minimum outage probability and optimal power allocation for fading multiple-access channels, in: IEEE International Symposium on Information Theory (ISIT), June 2000, p. 305

[11] G. Caire, G. Taricco, E. Biglieri, Optimum power control over fading channels, IEEE Trans. Inf. Theory 45 (5) (July 1999) 1468-1489.

[12] M.D. McDonnell, D. Abbott, What is stochastic resonance? Definitions, misconceptions, debates, and its relevance to biology, PLoS Comput. Biol. 5 (5) (May 2009) $1-9$.

[13] L. Gammaitoni, P. Hanggi, P. Jung, F. Marchesoni, Stochastic resonance, Rev. Mod. Phys. 70 (1) (Jan. 1998) 223-287.

[14] H. Chen, P.K. Varshney, S.M. Kay, J.H. Michels, Theory of the stochastic resonance effect in signal detection: Part I-Fixed detectors, IEEE Trans. Signal Process. 55 (7) (July 2007) 3172-3184

[15] H. Chen, P.K. Varshney, J.H. Michels, Noise enhanced parameter estimation, IEEE Trans. Signal Process. 56 (10) (Oct. 2008) 5074-5081.

[16] A. Patel, B. Kosko, Optimal noise benefits in Neyman-Pearson and inequalityconstrained signal detection, IEEE Trans. Signal Process. 57 (5) (May 2009) $1655-1669$
[17] S. Bayram, S. Gezici, Noise enhanced M-ary composite hypothesis-testing in the presence of partial prior information, IEEE Trans. Signal Process. 59 (3) (Mar. 2011) 1292-1297.

[18] S. Bayram, S. Gezici, H.V. Poor, Noise enhanced hypothesis-testing in the restricted Bayesian framework, IEEE Trans. Signal Process. 58 (8) (Aug. 2010) 3972-3989.

[19] C. Goken, S. Gezici, O. Arikan, Optimal stochastic signaling for powerconstrained binary communications systems, IEEE Trans. Wirel. Commun. 9 (12) (Dec. 2010) 3650-3661.

[20] C. Goken, S. Gezici, O. Arikan, Optimal signaling and detector design for powerconstrained binary communications systems over non-Gaussian channels, IEEE Commun. Lett. 14 (2) (Feb. 2010) 100-102.

[21] B. Dulek, S. Gezici, Detector randomization and stochastic signaling for minimum probability of error receivers, IEEE Trans. Commun. 60 (4) (Apr. 2012) 923-928.

[22] H. Chen, P.K. Varshney, S.M. Kay, J.H. Michels, Theory of the stochastic resonance effect in signal detection: Part II-Variable detectors, IEEE Trans. Signal Process. 56 (10) (Oct. 2007) 5031-5041.

[23] B. Dulek, S. Gezici, Optimal stochastic signal design and detector randomization in the Neyman-Pearson framework, in: 37th IEEE Int. Conf. Acous., Speech, Signal Process. (ICASSP), Kyoto, Japan, Mar. 25-30, 2012, pp. 3025-3028.

[24] E.L. Lehmann, Testing Statistical Hypotheses, 2nd ed., Chapman \& Hall, New York, 1986

[25] M. Azizoglu, Convexity properties in binary detection problems, IEEE Trans. Inf. Theory 42 (4) (July 1996) 1316-1321.

[26] B. Dulek, S. Gezici, O. Arikan, Convexity properties of detection probability under additive Gaussian noise: optimal signaling and jamming strategies, IEEE Trans. Signal Process. 61 (13) (July 2013) 3303-3310.

[27] S. Bayram, N.D. Vanli, B. Dulek, I. Sezer, S. Gezici, Optimum power allocation for average power constrained jammers in the presence of non-Gaussian noise, IEEE Commun. Lett. 16 (8) (Aug. 2012) 1153-1156.

[28] B. Dulek, M.E. Tutay, S. Gezici, P.K. Varshney, Optimal signaling and detector design for M-ary communication systems in the presence of multiple additive noise channels, Digit. Signal Process. 26 (Mar. 2014) 153-168.

[29] M.F. Keskin, M.N. Kurt, M.E. Tutay, S. Gezici, O. Arikan, Maximization of average number of correctly received symbols over multiple channels in the presence of idle periods, Digit. Signal Process. 54 (C) (July 2016) 95-118.

[30] A.D. Sezer, S. Gezici, H. Inaltekin, Optimal channel switching strategy for average capacity maximization, IEEE Trans. Commun. 63 (6) (June 2015) 2143-2157.

[31] A.D. Sezer, S. Gezici, Average capacity maximization via channel switching in the presence of additive white gaussian noise channels and switching delays, IEEE Trans. Wirel. Commun. (2016)

[32] A. Mesodiakaki, F. Adelantado, L. Alonso, C. Verikoukis, Energy efficiency analysis of secondary networks in cognitive radio systems, in: IEEE International Conference on Communications (ICC), June 2013, pp. 4115-4119.

[33] A. Mesodiakaki, F. Adelantado, L. Alonso, C. Verikoukis, Performance analysis of a cognitive radio contention-aware channel selection algorithm, IEEE Trans. Veh. Technol. 64 (5) (May 2015) 1958-1972.

34] T.M. Cover, J.A. Thomas, Elements of Information Theory, Wiley-Interscience, 1991.

[35] S. Boyd, L. Vandenberghe, Convex Optimization, Cambridge University Press Cambridge, UK, 2004

[36] R.T. Rockafellar, Convex Analysis, Princeton University Press, Princeton, NJ, 1968.

[37] D.P. Bertsekas, A. Nedic, A.E. Ozdaglar, Convex Analysis and Optimization, Athena Specific, Boston, MA, 2003.

Ahmet Dundar Sezer was born in 1989 in Emet, Kutahya, Turkey. He received both his B.S. and M.S. degrees in Electrical and Electronics Engineering from Bilkent University, Ankara, Turkey, in 2011 and 2013, respectively. He is currently working towards the Ph.D. degree at Bilkent University. His current research interests include signal processing, wireless communications, and optimization.

Sinan Gezici received the B.S. degree from Bilkent University, Turkey in 2001, and the Ph.D. degree in Electrical Engineering from Princeton University in 2006. From 2006 to 2007, he worked at Mitsubishi Electric Research Laboratories, Cambridge, MA. Since 2007, he has been with the Department of Electrical and Electronics Engineering at Bilkent University, where he is currently an Associate Professor. Dr. Gezici's research interests are in the areas of detection and estimation theory, wireless communications, and localization systems. Among his publications in these areas is the book Ultra-wideband Positioning Systems: Theoretical Limits, Ranging Algorithms, and Protocols (Cambridge University Press, 2008) Dr. Gezici is an associate editor for IEEE Transactions on Communications IEEE Wireless Communications Letters, and Journal of Communications and Networks. 